\title{
Influencia de la relación agua/cemento en la elaboración del mortero normalizado de los cementos con puzolana
}

\section{Influence of the relationship water/cement upon the processing of cements with pozzolana in standard mortar}

Fecha de recepción: 14-XI-00

Fecha de aceptación: 25-V-01
M. GENER RIZO,J. LUIS ÁLVAREZ CABRERA Centro Técnico para el Desarrollo de los Materiales de Construcción

CUBA

\section{RESUMEN}

La elaboración del mortero normalizado se realiza internacionalmente por diferentes métodos, pero existen dos tendencias fundamentales, la enunciada por ISO y por ASTM. La norma cubana de ensayos fisico-mecánicos de cemento se basa en la norma ISO, por lo que para la elaboración del mortero normalizado se utiliza una relación agua/cemento constante. Esto ha provocado discrepancias con los usuarios del cemento, especialmente cuando se ensayan los cementos que contienen puzolanas, ya que se plantea que no se tiene en cuenta la mayor demanda de agua de estos materiales.

En el presente trabajo se presenta un estudio del comportamiento de cementos Portland Puzolánicos (PP-250) elaborados con una relación agua/ cemento fija y variable, lograda a partir de la fluidez de la pasta de cemento Portland puro (P-350).

Los resultados obtenidos indican que se producen disminuciones en la resistencia mecánica en los morteros de cemento PP-250 elaborados con agual cemento variable y recomienda la adopción de un procedimiento opcional para elaborar el mortero de calidad de los cementos con adiciones de puzolana.

\section{SUMMARY}

The processing of standard mortar is completed following different methods in accordance with the country, but they exist two fundamental tendecies, the ISO and the ASTM. The cuban norm for mechanic-physic tests is based in ISO, and so they use a constant relationship water/cement in the processing of standard mortar a great problem concerning the cement users when they tested those mixed with puzzolanes, because they don't take care of the bigger water needs of those materials.

In this work we present an study of the behaviour of Pozzolanic Portland cements (PP-250) elaborates with a fix and changeable relationship water/cement, obtained starting from the fluidity of the pure Portland cement. (P-350)

The results obtained shows that the mechanical resistance decreased in cement mortars $P P-250$ realised with changeable relationship water/cement. So we recommend the adoption of an optional procedure to elaborate a quality mortar with pozzolana cements.

\section{INTRODUCCIÓN}

La producción de cemento a nivel internacional, al igual que el resto de las industrias, se ha caracterizado por la disminución de sus gastos de producción a través del desarrollo de nuevos procedimientos tecnológicos que le han permitido reducir el consumo energético en el proceso y racionalizar lo más posible los gastos indirectos asociados al mismo.
En términos energéticos, una de las vías más universales empleadas en la industria del cemento, lo constituye el empleo de las adiciones activas incluidas en el cemento. Las cuales son añadidas al clínkery molidas conjuntamente con éste.

En la actualidad la producción de cemento a nivel mundial está dirigida a la fabricación de diferentes tipos y calidades de cementos, con el objetivo de satisfacer los 
requerimientos de los diversos elementos de hormigón y de las obras en general además de consideraciones de ahorro energético. Según información de CEMBUREAU, en 1996 la producción de cemento con adiciones en Europa de calidades de 32,5 Mpa y 42,5 Mpa, alcanzó el $94 \%$ del total de la producción (1).

La determinación de la resistencia mecánica de los cementos constituye uno de los parámetros fundamentales para la determinación de su calidad, no sólo por lo que representa el valor absoluto especificado, sino por el conocimiento de lo que significa para su correcto empleo en las diferentes calidades del hormigón y en los usos específicos para las diversas etapas constructivas.

La elaboración del mortero de calidad de cemento se realiza, en los diferentes países, por diversos procedimientos, aunque existen internacionalmente dos tendencias predominantes: la enunciada por la Internacional Organización for Standarization (ISO) y porla American Society for Testing and Materials(ASTM).

También se emplean las metodología de las normas Británicas y otras propias de algunos países que recogen aspectos de las dos primeras. A pesar de los esfuerzos realizados para establecer una metodología única (2), se informa que 31 países adoptan las normas ISO, 16 las ASTM, 19 las Británicas y 10 emplean normas propias (3), además entre los mayores productores de cementos, cerca del $50 \%$ utilizan las ISO (4).

El incremento progresivo de la producción de los cementos con adiciones en Cuba, la necesidad de la integración al mercado nacional e internacional y la satisfacción de los requerimientos del cliente, exigen de documentos normativos que permitan avalar las características de este producto y los procedimientos de ensayos para su evaluación (5).

Uno de los aspectos más discutidos en nuestro país es el relacionado con la elaboración del mortero normalizado en los cementos con puzolanas, dado que se emplea una relación agua/cemento constante y, por lo tanto, "no tiene en cuenta la mayor demanda de agua del material puzolánico".

\subsection{Antecedentes}

Como se había enunciado anteriormente, para la ejecución de los ensayos de cemento existen en el mundo dos tendencias fundamentales: la ISO (6) y la ASTM (7). Las diferencias principales en cuanto a la elaboración del mortero normalizado para determinar la resistencia mecánica, se muestran en la Tabla 1.

La norma cubana NC 54-207: 88 (8) se basa en el método ISO, y fue aprobado a principios de los años 70. En aquel momento se adquirió el equipamiento necesario y se realizaron los estudios correspondientes con diferentes arenas silíceas, para encontrar una que, por sus características, pudiera ser homologada con la arena Belga, que es la que se toma como referencia para los países que emplean el procedimiento ISO. Por aquel entonces, en Cuba, prácticamente, sólo se producía cemento Portland puro.

Como parte de los trabajos del Comité Técnico de Normalización de Cemento (CTN-22), se encuentra la revisión de la norma NC 54-207 (Cemento Ensayos físico-mecánicos), que, al fundamentarse en la metodología ISO, establece una relación agua/cemento igual a 0,5 para todos los tipos de cemento; es precisamente en este ensayo donde radican una de las mayores diferencias con relación a la norma ASTM.

Teniendo en cuenta el incremento en Cuba de la producción de diferentes tipos y calidades de cementos mezclados con puzolana y conociendo que, en general, estas adiciones demandan una mayor cantidad de agua al elaborar hormigones de determinada consistencia, se hace necesario estudiar la influencia de la adición de agua en la determinación de la resistencia del mortero normalizado de cemento. Sobre la base de estos resultados se recomendaría -o no-, incluir la adopción de un ensayo alternativo con relación agua/cemento diferente de 0,5 en la futura norma de ensayos físico-mecánicos de resistencia a flexión y compresión.

Entre los trabajos realizados anteriormente se encuentran Ruiz(9), donde se plantea que en cementos elaborados en el laboratorio (molino $100 \mathrm{~kg} / \mathrm{h}$ ) con adiciones de puzolana natural de 10,15 y $20 \%$ en peso, se obtuvieron valores de la relación agua/cemento de $0.52,0,55$ y 0,57 respectivamente. Las pérdidas de resistencia a la compresión a los 3 días fueron de $0,4 \mathrm{Mpa}, 1,50 \mathrm{Mpa}$ y $2,25 \mathrm{Mpa}$ respectivamente, con respecto a los mismos cementos ensayados a una relación $\mathrm{a} / \mathrm{c}=0,5$. A los 28 días se obtuvieron semejantes valores de resistencia para todos los cementos.

Rabilero (10) realizó ensayos comparativos de resistencia a la compresión a 20 muestras de cemento PP-250 producidos en la Empresa José Mercerón, con una adición promedio de toba del 10,25\%, los morteros fueron elaborados con $225 \mathrm{ml}$ de agua $(\mathrm{a} / \mathrm{c}=0,5)$ y con una adición media de agua de $239 \mathrm{ml}$ para obtener la fluidez del cemento $\mathrm{P}-350$ producido en dicha Empresa. Esto representó una relación a/c de 0,53 . Los valores medios de resistencia a la compresión obtenidos a los 7 días fueron de 35,8 Mpa para $225 \mathrm{ml}$ de agua y de 32,7 Mpa con $239 \mathrm{ml}$. A los 28 días se obtuvieron resistencias de 46,2 y 42,4 Mpa respectivamente.

Gener (11) para cementos elaborados con un mismo clínkery adiciones del 10 y $15 \%$ de diferentes yacimien- 
TABLA 1

Diferencias fundamentales entre Norma ISO y ASTM

\begin{tabular}{|l|c|c|}
\hline \multicolumn{1}{|c|}{ ISPECTOS } & ISO & ISTH \\
\hline Relación agua/ cemento & 0.5 para todo tipo de cemento & $\begin{array}{c}0.485 \text { Portland puro } \\
\text { Variable para cementos } \\
\text { mezclados con fluidez } 110 \pm 5 \%\end{array}$ \\
\hline Granulometria de la arena & $0.08-2.00 \mathrm{~mm}$ & $0.15-0.6 \mathrm{~mm}$ \\
\hline Compactación & Mecánica & Manual \\
\hline Temperatura de Laboratorio & $20 \pm 2^{\circ} \mathrm{C}$ & $\begin{array}{c}20^{\circ} \mathrm{C} \text { ó } 27.5^{\circ} \mathrm{C} \\
\text { cámara húmeda } 23 \pm 1.7^{\circ} \mathrm{C}\end{array}$ \\
\hline Moldes & $\begin{array}{c}\text { Moldes prismáticos de } \\
40 \times 40 \times 160 \mathrm{~mm}\end{array}$ & $\begin{array}{c}\text { Moldes cúbicos de } 50 \mathrm{~mm}, \\
\text { se permite moldes de } \\
40 \times 40 \times 160 \mathrm{~mm} \\
\text { por C } 349-95\end{array}$ \\
\hline
\end{tabular}

tos de puzolana en morteros con relación agua/cemento de 0,5 , obtuvo pérdidas de fluidez del 3 al $12 \%$ para el 10 $\%$ de adición y del 7 al $20 \%$ para el $15 \%$. Esto se le atribuyó a la influencia de la composición mineralógica de las puzolanas en el requerimiento de agua del mortero.

Los resultados obtenidos por Alonso (12) en morteros de cemento preparados en el laboratorio con adiciones de $8,10,12$ y $15 \%$ de puzolana natural y elaborados según el procedimiento de la norma ASTM, aportan valores de relación a/c de $0,51,0,53,0,54$ y 0,55 respectivamente.

Es conveniente señalar que la determinación de la resistencia en morteros y hormigones está sujeta a la influencia de numerosas variables tales como: materias primas, finura del cemento y, en especial, su composición granulométrica, contenido y tipo de adición, composición mineralógica y propiedades físicas de las adiciones, áridos empleados, temperatura, humedad y curado, entre otros.

\section{PARTE EXPERIMENTAL}

\subsection{Materiales y procedimientos de trabajo}

\subsubsection{Materiales}

Para la evaluación de las propiedades físico mecánicas de los cementos se diseñó un muestreo en tres fábricas de cemento representativas de la totalidad de las existentes en Cuba, y se consideró necesario realizar, además, estudios de algunas propiedades químicas que permitieran una caracterización completa de las muestras objeto de análisis.

Se recogieron muestras de tobas y de yesos necesarias para la estimación del contenido de puzolana en los cementos, las cuales fueron caracterizadas mediante análisis químico y mineralógico.

\subsubsection{Procedimiento de Trabajo}

\subsubsection{Ensayos realizados}

Las características químicas y físico-mecánicas de los cementos fueron determinadas por los procedimientos establecidos en las normas cubanas NC 54-203:79(13) y NC 54-207:88 (8).

Los ensayos realizados se corresponden con algunos de los requisitos que se regulan en las normas de especificaciones de calidad para los cementos Portland P-350 ( clínker + yeso mineral) y Portland Puzolánico PP-250 ( clínker + yeso mineral + puzolana entre 6 y 20 $\%)$. Estos fueron: residuo insoluble, pérdida por calcinación, trióxido de azufre, superficie específica Blaine, finura en el tamiz de 0,090 $\mathrm{mm}$, peso específico, consistencia normal y resistencia a la flexión y compresión a los 3, 7 y 28 días. Como ensayos adicionales se incluyeron: estimación del contenido de puzolana en los cementos y fluidez a los morteros.

En la elaboración del mortero de cemento para la determinación de la resistencia a flexión-compresión, se procedió de la siguiente forma:

. Elaboración del mortero con los cementos P-350 según NC 54-207: 88, adicionalmente medir la fluidez en la mesa de sacudidas.

- Elaboración del mortero con los cementos PP-250 según NC 54-207: 88 y medir la fluidez.

. Elaboración del mortero con los cementos PP 250 con una fluidez semejante a la obtenida con lo s cementos P-350. 
. Elaboración del mortero con cementos P-350 y P-250 seleccionados (4 muestras) con una fluidez $105 \pm 5 \mathrm{~mm}$.

La obtención de los cementos de la fábricas seleccionadas contempló la ubicación de las mismas. La fabrica A es la suministradora fundamental de la región occidental del país, incluyendo la capital; las fabricas $\mathrm{C}$ y $\mathrm{S}$ suministran cemento a las regiones central y occidental. También se consideró las calidades de las tobas empleadas en dichas fábricas que comprenden desde la menos activa, hasta la de mayor actividad, o sea representan las diferentes composiciones mineralógicas de las tobas existentes en el país.

\section{RESULTADOS OBTENIDOS}

\subsection{Puzolana y yeso}

La composición mineralógica de las puzolanas se realizó mediante la técnica de Difracción de Rayos X (DRX) y Análisis Térmico Diferencial. Mediante los análisis químicos se determinaron el residuo insoluble y la pérdida por ignición,Tablas 2 y 3 .

En general, las puzolanas están constituidas por una estructura zeolítica del tipo mordenita o mordenita-clinoptilolita, plagioclasa, pequeñas cantidades de calcita, montmorillonita, y abundante cuarzo en la puzolana Siguaney, además de una fase vítrea dada en DRX por la banda difusa entre los $22-32^{\circ}$, valores de $2 \theta$.
El método empleado para la determinación de la actividad puzolánica se corresponde con uno físico-mecánico desarrollado en el Instituto de Ciencias de la Construcción Eduardo Torroja (14), el mismo se basa en la determinación de la resistencia a la compresión en un mortero preparado con una parte de cal y tres de puzolana, sometido a un ambiente de vapor de agua durante 72 horas. Establece que cuando la resistencia obtenida es menor de $4 \mathrm{Mpa}$, el material no es activo; valores entre 4 Mpa-7 Mpa tiene actividad media y con valores superiores a $10 \mathrm{Mpa}$ la actividad es alta. Los valores de los residuos insolubles y pérdidas por ignición están dentro del intervalo para estos yacimientos. La menor actividad puzolánica corresponde a la toba Los Congos y, la mayor, a la toba Carolina.. El contenido de $\mathrm{SO}_{3}$ en los yesos esta entre 28,0 y $39,13 \%$.

\subsection{Estudio de las características de los cementos}

En las tablas 4 y 5 se presentan los resultados de los valores medios de los ensayos realizados a las muestras de los cementos P-350 y PP-250 objeto de análisis.

Los valores de fluidez, para mayor comprensión, están expresados, en todo el trabajo, como el radio del esparcimiento de la masa del mortero, en lugar del valor del diámetro $(\mathrm{d}=2 \mathrm{r}$, en $\mathrm{mm})$.

Los cementos P- 350 cumplieron con los parámetros de calidad establecidos para esta marca en la norma NC 54-205: 80. Para el caso de los cementos P-350, el

TABLA2

Resumen de la composición mineralógica de las tobas

\begin{tabular}{|c|c|c|c|}
\hline $\begin{array}{c}\text { Fasel contenido } \\
\text { estimado }\end{array}$ & $\begin{array}{c}\text { Los Congos } \\
\text { Tábrica A }\end{array}$ & $\begin{array}{c}\text { Carolina } \\
\text { Fábrica C }\end{array}$ & $\begin{array}{c}\text { Siguaney } \\
\text { Fábrica S }\end{array}$ \\
\hline Vítrea & Poca & Abundante & Moderada \\
\hline Zeolita & Moderada & Moderada & Moderada \\
\hline Cuarzo & - & - & Abundante \\
\hline Plagioclasa & Moderada & Poca & Moderada \\
\hline Calcita & Poca & Moderada & Poca \\
\hline Montmorillonita & Moderada & Poca & Moderada \\
\hline
\end{tabular}

TABLA 3

Características químicas de las tobas y los yesos

\begin{tabular}{|c|c|c|c|c|c|}
\hline \multicolumn{2}{|c|}{ lábrica } & $\mathrm{SO} \mathrm{s}(\%)$ & RI $(\%)$ & $\mathrm{PPI}(\%)$ & $(4 c t .9 u z$ \\
\hline \multirow{2}{*}{ A } & Toba & - & 34.67 & 7.45 & 7.3 \\
\hline & Yeso & 28.00 & 21.73 & 9.55 & - \\
\hline \multirow{2}{*}{$\mathrm{C}$} & Toba & - & 26.15 & 18.32 & 13.8 \\
\hline & Yeso & - & - & - & - \\
\hline \multirow{2}{*}{$S$} & Toba & - & 62.04 & 8.98 & 8.7 \\
\hline & Yeso & 39.13 & 13.33 & 11.47 & - \\
\hline
\end{tabular}


TABLA 4

Valores medios de las características de los cementos P-350

\begin{tabular}{|c|c|c|c|c|c|c|}
\hline \multirow{2}{*}{\multicolumn{2}{|c|}{ Características }} & \multirow{2}{*}{ UM } & \multirow{2}{*}{ NC54-205: 80} & \multicolumn{3}{|c|}{ Fábricas } \\
\hline & & & & A & $4, \mathbf{C}$ & $\mathbf{S}$ \\
\hline \multicolumn{2}{|c|}{$\mathrm{RI}$} & $\%$ & 3.0 máximo & 2.95 & 1.55 & 0.97 \\
\hline \multicolumn{2}{|c|}{ PPI } & $\%$ & 3.5 máximo & 1.84 & 1.72 & 2.02 \\
\hline \multicolumn{2}{|c|}{$\mathrm{SO}_{3}$} & $\%$ & 3.5 máximo & 2.53 & 2.88 & 1.79 \\
\hline \multicolumn{2}{|c|}{ Peso especifico } & $\mathrm{g} / \mathrm{cm}^{3}$ & - & 3.12 & 3.15 & 3.13 \\
\hline \multicolumn{2}{|c|}{ S. E. Blaine } & $\mathrm{cm}^{2} / \mathrm{g}$ & 2800 mínimo & 3383 & 3324 & 2991 \\
\hline \multicolumn{2}{|c|}{ Finura $0,009 \mathrm{~mm}$} & $\%$ & 10 máximo & 8 & 3.4 & 7.19 \\
\hline \multicolumn{2}{|c|}{$\mathrm{CN}$} & $\%$ & - & 24 & 24 & 23 \\
\hline \multicolumn{2}{|c|}{ Fluidez } & $\mathrm{mm}$ & - & 67 & 69 & 69 \\
\hline \multirow{3}{*}{$\mathrm{Rf}$} & $3 \mathrm{~d}$ & $\mathrm{MPa}$ & 3.0 mínimo & 5.1 & 4.69 & 3.85 \\
\hline & $7 \mathrm{~d}$ & $\mathrm{MPa}$ & 4.0 minimo & 6.0 & 5.92 & 5.15 \\
\hline & $28 \mathrm{~d}$ & $\mathrm{MPa}$ & 6.0 mínimo & 7.6 & 7.58 & 6.94 \\
\hline \multirow{3}{*}{$\mathrm{Rc}$} & $3 \mathrm{~d}$ & $\mathrm{MPa}$ & 17.0 mínimo & 22.4 & 24.96 & 23.65 \\
\hline & $7 \mathrm{~d}$ & $\mathrm{MPa}$ & 25.0 mínimo & 30.0 & 31.72 & 31.01 \\
\hline & $28 \mathrm{~d}$ & $\mathrm{MPa}$ & 35.0 mínimo & 39.5 & 42.49 & 40.94 \\
\hline \multicolumn{4}{|c|}{ Cantidad de muestras } & 20 & 19 & 20 \\
\hline
\end{tabular}

TABLA5

Valores medios de las características de los cementos PP-250

\begin{tabular}{|c|c|c|c|c|c|c|}
\hline \multirow{2}{*}{\multicolumn{2}{|c|}{ Garacteristicas. }} & \multirow{2}{*}{ UM } & \multirow{2}{*}{ Ne $54.216: 80$} & \multicolumn{3}{|c|}{ CHMUNLOS/ Fabricas } \\
\hline & & & & A 1.9 & 181 & 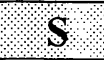 \\
\hline \multicolumn{2}{|l|}{$\mathrm{RI}$} & $\%$ & & 8.77 & 5.67 & 9.37 \\
\hline \multicolumn{2}{|c|}{ PPI } & $\%$ & & 4.82 & 2.83 & 4.91 \\
\hline \multicolumn{2}{|c|}{$\mathrm{SO}_{3}$} & $\%$ & 2.5 máximo & 2.38 & 2.81 & 2.05 \\
\hline \multicolumn{2}{|c|}{$\mathrm{CN}$} & $\%$ & & 29 & 26 & 26 \\
\hline \multicolumn{2}{|c|}{ Contenido de toba } & $\%$ & & 15.02 & 16.90 & 15,08 \\
\hline \multicolumn{2}{|c|}{$\mathrm{a} / \mathrm{c}$} & - & & 0.5 & 0.5 & 0.5 \\
\hline \multicolumn{2}{|c|}{ Fluidez } & $\mathrm{mm}$ & & 60 & 62 & 61 \\
\hline \multirow{3}{*}{$\mathrm{Rc}$} & $3 \mathrm{~d}$ & $\mathrm{MPa}$ & & 20.1 & 20.3 & 14.9 \\
\hline & $7 \mathrm{~d}$ & $\mathrm{MPa}$ & 3.0 mínimo & 26.5 & 26.3 & 22.3 \\
\hline & $28 \mathrm{~d}$ & $\mathrm{MPa}$ & 5.0 mínimo & 37.2 & 39.5 & 33.6 \\
\hline \multicolumn{2}{|c|}{ Fluidez } & $\mathrm{mm}$ & & 68 & 69 & 70 \\
\hline \multicolumn{2}{|c|}{ c. agua } & $\mathrm{ml}$ & & 240 & 237 & 234 \\
\hline \multicolumn{2}{|c|}{$\mathrm{a} / \mathrm{c}$} & - & & 0.53 & 0.53 & 0.52 \\
\hline \multirow{3}{*}{ Rc } & $3 \mathrm{~d}$ & $\mathrm{MPa}$ & & 17.5 & 16.5 & 10.7 \\
\hline & $7 \mathrm{~d}$ & $\mathrm{MPa}$ & 17.0 mínimo & 23.9 & 23.2 & 19.1 \\
\hline & $28 \mathrm{~d}$ & $\mathrm{MPa}$ & 25.0 mínimo & 35.3 & 36.5 & 30.4 \\
\hline \multicolumn{4}{|c|}{ Cantidad de muestras } & 25 & 21 & 23 \\
\hline
\end{tabular}


mortero de calidad fue preparado según la norma cubana NC 54-207: 88, esto es, con una cantidad de agua de $225 \mathrm{ml}$ para una relación agua cemento de 0,5 . Se midió la fluidez y se obtuvo un valor medio por fábrica entre 67 y $69 \mathrm{~mm}$. En los cementos PP-250 se preparó una serie tal y como se establece en la norma de referencia, se midió la fluidez y se obtuvo un valor medio por fábrica entre 60-62 mm; en una segunda serie se elaboró el mortero con la cantidad de agua necesaria para lograr la fluidez de $69 \pm 2 \mathrm{~mm}$ (P-350) y, de esta forma, se obtuvieron relaciones agua cemento entre 0,52 y 0,53 .

Los cementos PP-250 también cumplieron en general con los requisitos de la norma NC 54-216: 80. Para contenidos de toba entre un $15-17 \%$ se obtuvieron diferentes valores en los residuos insolubles de los cementos, lo cual se relaciona con las diferencias en la composición mineralógica de estos materiales $\mathrm{y}$, por lo tanto, con sus residuos insolubles.

Para los morteros elaborados con una fluidez $69 \pm$ $2 \mathrm{~mm}$ y con relación agua/cemento entre $0,52-0,53$, se obtuvieron consumos adicionales de agua entre un 4-6\%, con disminuciones en la resistencia a la compresión a los 3 días entre un 13-25\% y a los 28 días entre 5-10\% al compararlos con los obtenidos en los morteros elaborados con una relación $\mathrm{a} / \mathrm{c}=0,5$.

Se determinó si existía diferencia significativa en los valores de resistencia a la compresión en los morteros de cemento PP-250 elaborados con una relación a/c fija e igual a 0,5 y los elaborados con una fluidez constante y por tanto a una relación a/ c variable.

Se obtuvo una diferencia significativa entre los valores de resistencia a la compresión en los morteros de cemento elaborados a una relación a/c de 0,5 y entre $0,52-0,53$ para un nivel de confiabilidad del $95 \%$, también existieron diferencias para los valores de la fluidez.

\subsection{Estudio de morteros de cemento elaborados con una fluidez $105 \pm 5 \mathrm{~mm}$.}

Se realizaron ensayos físico-mecánicos sobre probetas prismáticas de $40 \times 40 \times 160 \mathrm{~mm}$ de morteros de cemento confeccionadas con la cantidad de agua requerida para lograr una fluidez de $105 \pm 5 \mathrm{~mm}, \mathrm{y}$, de este modo, poder evaluar la influencia de la adición de puzolana en los requerimientos de agua de un mortero fluido. Esto permite conocer de un modo más preciso las propiedades mecánicas de los cementos con relación a su ulterior comportamiento en hormigones (Tabla 6).

Para la elaboración de los morteros con esta fluidez, se obtuvo un consumo adicional de agua de 9-17\%. Para el cemento $\mathrm{P}-350$ las pérdidas de resistencia a la compresión fueron del orden del $13 \%$ a los 3 días, al compararlas con los obtenidos con una relación a/c de 0,5 ; para los cementos mezclados, a esta misma edad, disminuyeron aproximadamente el $30 \%$.

A los 28 días de endurecimiento las pérdidas fueron del $10 \%$ en el cemento $\mathrm{P}-350 \mathrm{y}$ del $14 \%$ aproximadamente en los PP-250.

\section{CONCLUSIONES}

Los cementos P- 350 y PP- 250 estudiados cumplieron en general con los requisitos de calidad establecidos en las normas cubanas correspondientes.

El valor de la fluidez para los morteros de cemento P-350 elaborados por la norma cubana, relación agua/ cemento igual a 0,5 , fue de $134-138 \mathrm{~mm}(\mathrm{r}=68 \pm 1 \mathrm{~mm})$ y el valor medio para los PP- 250 fue de $120-124 \mathrm{~mm}$ $(\mathrm{r}=61 \pm 1 \mathrm{~mm})$.

Para lograr la fluidez de $69 \pm 2 \mathrm{~mm}$ en los morteros de cementos mezclados fue necesario añadir una mayor

TABLA 6

Morteros de cemento elaborados con una fluidez $105 \pm 5 \mathrm{~mm}$

\begin{tabular}{|c|c|c|c|c|c|c|c|}
\hline \multirow{2}{*}{\multicolumn{2}{|c|}{61159410}} & \multicolumn{2}{|c|}{ Habrien } & \multicolumn{2}{|c|}{ Habricen } & \multicolumn{2}{|c|}{ 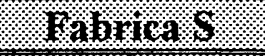 } \\
\hline & & 18.50 & $101 / 25$ & 14350 & $8 \mathrm{P} / 2,51$ & $1 / 350$ & $181 / 250$ \\
\hline \multicolumn{2}{|l|}{ Agua (ml) } & 2.45 & 252 & 2.47 & 260 & 246 & 262 \\
\hline \multicolumn{2}{|l|}{$\mathrm{a} / \mathrm{c}$} & 0.544 & 0.56 & 0.548 & 0.57 & 0.546 & 0.58 \\
\hline \multicolumn{2}{|l|}{ Fluidez } & 106 & 106 & 105 & 107 & 105 & 101 \\
\hline \multirow{3}{*}{$\mathrm{Rf}(\mathrm{MPa})$} & 3 días & 4.63 & 3.96 & 3.18 & 2.48 & 2.89 & 2.46 \\
\hline & 7 días & 5.35 & 5.11 & 4.37 & 3.87 & 4.19 & 3.09 \\
\hline & 28 días & 7.21 & 6.57 & 5.92 & 4.71 & 5.99 & 4.86 \\
\hline \multirow{3}{*}{$\mathrm{Rc}(\mathrm{MPa})$} & 3 días & 18.80 & 17.3 & 22.43 & 14.1 & 20.36 & 11.01 \\
\hline & 7 días & 26.90 & 24.7 & 27.81 & 21.5 & 26.65 & 18.01 \\
\hline & 28 días & 38.30 & 35.7 & 36.63 & 32.4 & 38.14 & 28.81 \\
\hline
\end{tabular}


cantidad de agua y se obtuvo una relación agua/cemento entre 0,52-0,53. Esto provocó una caída de la resistencia a la compresión del orden de $25 \%$ a los 3 días y del $10 \%$ a los 28 días de endurecimiento.
Se recomienda la necesidad de adoptar un procedimiento opcional para elaborar el mortero normalizado de los cementos con puzolana a una fluidez constante y no con una relación agua/cemento constante.

\section{BIBLIOGRAFÍA}

(1) Gayoso, R. Cemento Portland normal y aditivo químico mineral en hormigones de alta resistencia y durabilidad. Ponencia IV Conferencia Científico Técnico de la Construcción. C. Habana, Cuba, abril 2000, pp. 173

(2) Dutron, P. Rapportintroductif an $4^{\mathrm{eme}}$ seminaire bases scientifiques des specifications pous le ciment et le developpment des methodes d'essais. $8^{\text {vo }}$ Congresso International de Química do Cimento, Río Janeiro,. Vol. VI. 1986, pp 1399-1413.

(3) Sanjurjo, R. Trabajo presentado en la mesa redonda "Cementos con adiciones" CTDMC. C. Habana, Cuba, octubre 1999.

(4) $\mathrm{Da}$ Costa, H. Resistencia a compresión de cementos por tres diferentes métodos de ensayos. $3^{\text {er }}$ Congreso Brasileiro del cemento, ABCP, Vol. 1, 1993, pp 23-42.

(5) Gener, M;. Santos, N y otros. Actualización de la Base Normativa de Cemento. Etapa 03 del PCT 02.12 “Complemento al estudio de los cementos mezclados". Informe CTDMC, C. Habana, Cuba, nov. 1999.

(6) ISO 679/ EN 196.1: 96. Métodos de ensayos de cemento. Determinación de la resistencia mecánica.

(7)C 109: 95. Método de ensayo para determinar la resistencia a la compresión en morteros de cemento hidráulico. ASTM V.04.01. 1997.

(8) NC 54- 207: 88 Materiales y productos de la construcción. Cemento. Ensayos físico-mecánicos.

(9) Ruiz, B. Determinación de la calidad de los cementos mezclados con diferentes dosificaciones de toba. III Congreso del Cemento y del Hormigón, C. Habana, Cuba, nov. 1996.

(10) Rabilero, A. Tésis Doctoral. Contribución al empleo de puzolanas naturales en la producción de cementos y otros aglomerantes. Santiago de Cuba, 1996.

(11) Gener, M; Alonso, J. Influencia de la composición mineralógica de puzolanas naturales en las propiedades de los cementos mezclados. Trabajo presentado en la III Conferencia Científica Técnica de la Construcción, C. Habana, Cuba, nov, 1997.

(12) Alonso, J. Ponencia presentada a la IV Conferencia Científico Técnica de la Construcción, C. Habana, Cuba, septiembre 1999.

NC 54- 203: 79 Materiales y productos de la construcción. Ensayos Químicos de cemento, clínker y pasta.

(13) Luxán, M. P. y otros. Estudio de la actitud de un material como adición activa al cemento. Rev. Materiales de Construcción. Vol. 35 , No 200, 1985. 